\title{
DETECÇÃO DE FALHAS POR MEIO ACÚSTICO UTILIZANDO ALGORITMO DE SELEÇÃO NEGATIVA
}

\section{FAULT DETECTION BY ACOUSTIC MEANS USING THE NEGATIVE SELECTION ALGORITHM}

Igor Feliciani Merizio ${ }^{1}$, Fábio Roberto Chavarette $^{1}$, Thiago Carreta Moro ${ }^{1}$, Roberto Outa ${ }^{2}$

${ }^{1}$ Universidade Estadual - UNESP. ${ }^{1}$ Faculdades de Tecnologia do Estado de São Paulo - FATEC, Araçatuba, SP.

E-mail: igorfeliciani@gmail.com.

RESUMO - Um sistema de monitoramento da integridade estrutural (SHM) avalia o estado de estruturas aeronáuticas, civil ou mecânicas e dá a previsão de sua vida remanescente, atuando na tomada de decisão, podendo intervir em situações críticas. Surgiu como uma viável alternativa econômica no monitoramento de estruturas, e na prevenção de falhas. Assim, esse sistema é definido como uma medida profilática, confiável e eficaz, contra a falha estrutural. Esse trabalho tem como objetivo o embasamento teórico e a detecção de falhas em tubos por meios acústicos, seguindo a norma ISO10534-1(1996) na coleta experimental. Esse método de detecção de falhas por via acústica necessita de consideravelmente menos dados de treinamento do que o usualmente utilizado na literatura, sendo aproximadamente $85 \%$ menos dados.

Palavras-chave: Monitoramento da Saúde Estrutural; Algoritmo de Seleção Negativa; Sistema Imunológico Artificial.

ABSTRACT - The System Integrity Monitoring (SHM) assesses the situation of aeronautical, civil or mechanical structures and provides the forecast of it is remaining life, acting in decision making, being able to intervene in critical situations. It has emerged as a viable economic alternative for monitoring structures and preventing failures. Thus, this system is defined as a prophylactic measure, reliable and effective against structural failure. This work has as objective the theoretical basis and the detection of failures in tubes by acoustic means, following the norm ISO10534-1 (1996) in the sampling. This method of fault detection using acoustic means requires considerably less training data than is usually used in the literature, with approximately $85 \%$ less data. 
Keywords: Structural Health Monitoring; Negative Selection Algorithm; Artificial Immune System.

\section{INTRODUÇÃO}

Estruturas aeronáuticas, civil ou mecânica estão sujeitas a falhas devido ao desgaste por fatores temporais, humanos e ambientais. Assim, as estruturas necessitam de uma determinada frequência de manutenção para que se evite falhas e apoio a prevenção de acidentes.

Atualmente, a indústria vem aumentando os investimentos em pesquisa e desenvolvimento de novas metodologias que atuem do diagnóstico da saúde de estruturas. Monitorando a saúde estrutural, evitando catástrofes e danos ambientais, humanitários e econômicos.

Recebe o nome de monitoramento da saúde estrutural (SHM - Structural Health Monitoring) a importante e atual linha de pesquisa que consiste na detecção de falhas em seu estado inicial para que se possa intervir na sua propagação e evitar prejuízos.

Um SHM deve atender, como destacado por Hall (1999), a aquisição e processamento de dados, validação e análise de sinais, identificação e caracterização de falhas, interpretação de mudanças adversas em uma estrutura e auxiliar a tomada de decisões.

A avaliação da integridade de estruturas mecânicas realizada por ensaios não-destrutivos como líquidos penetrantes, partículas magnéticas, ultrassonografia, radiografia, entre outras técnicas tradicionais, apesar de possuir bom aparato instrumental e serem diversamente empregas não satisfazem totalmente as crescentes necessidades industriais (Franco et al., 2009).

Estruturas mecânicas como tubos, por exemplo o Gasoduto Bolívia-Brasil, também conhecido como "Gasbol", precisam de constante manutenção e verificação de sua saúde estrutural.
A detecção de falhas e o monitoramento da integridade estrutural em tubos, utilizando meios acústicos, é ainda pouco pesquisado e empregado. Sendo o diferencial deste trabalho a utilização de uma onda sonora propagando no ar para a detecção de qualquer tipo de dano que pode comprometer o funcionamento de um tubo.

Na seção 3 mostra-se a base teórica para o desenvolvimento e programação de um SHM, na seção 4 os materiais utilizados e a montagem da bancada experimental, na seção 5 o desenvolvimento do projeto. Os resultados da pesquisa são exibidos e discutidos na seção 6 , sendo a seção 7 dedicada as conclusões.

\section{OBJETIVO DA PESQUISA}

O objetivo desse trabalho de pesquisa é desenvolver um SHMs para estruturas mecânicas utilizando técnicas do algoritmo de seleção negativa (ASN) (FORREST et al., 1994) para realizar a caracterização, analise e monitoramento da integridade estrutural de um tubo de impedância acústica partindo da coleta da pressão acústica em posições no interior do tubo baseando-se na norma ISO10534-1 (1996).

\section{FUNDAMENTAÇÃO TEORICA}

A base da técnica do algoritmo de seleção negativa (ASN) (Forrest et al., 1994) é a observação do reconhecimento de padrões exercido pelo sistema imunológico biológico no reconhecimento de linfócitos $T$, que ocorre no timo, para categorizar as células como benignas ou malignas. Divide-se o ASN em duas partes: Censoriamento e Monitoramento.

No Censoriamento ocorre a determinação de um conjunto de vetores próprios protegidos em paralelo com a geração de cadeias aleatórias para constituir 
a base-line após a avaliação da afinidade entre esses e os sinais próprios, de modo a rejeitar determinada cadeia que apresentar afinidade superior ao estipulado.

As Figuras 1 e 2 ilustram a fase de censoriamento e monitoramento do ASN.

Figura 1. Fluxograma da fase de censoriamento do ASN.

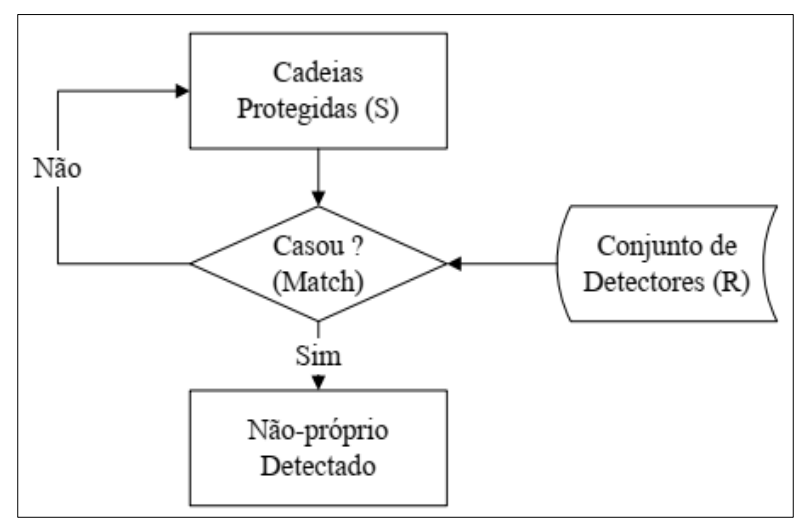

Fonte: (Castro, 2001).

No monitoramento, com a criação da base-line, avalia-se a afinidade entre essa e o conjunto de detectores. Caso a afinidade seja superior a um limiar estipulado um elemento não-próprio é identificado (De Castro, 2001).

Figura 2. Fluxograma da fase de monitoramento do ASN.

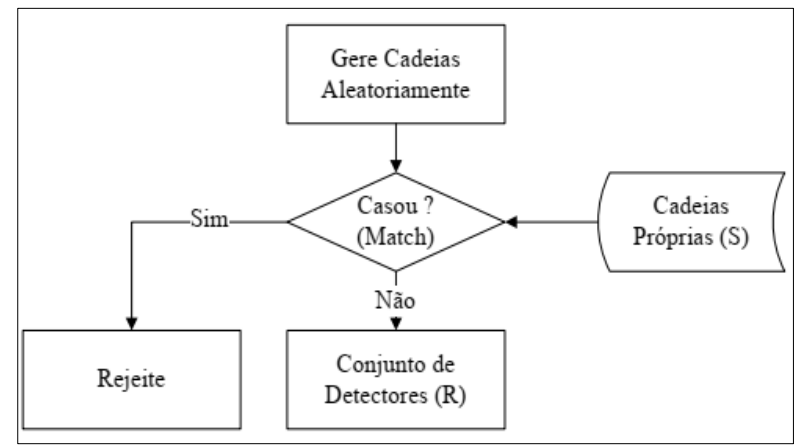

Fonte: (Castro, 2001).

Os detectores próprios são inicialmente definidos na fase de censoriamento, também chamados de cadeias próprias, esses representam a situação normal de operação do sistema. 0 conjunto de detectores, com a capacidade de reconhecer padrões não próprios, é gerado em sequência. Então, escolhe-se cadeias aleatórias, partindo da leitura dos dados. Os detectores operam como as células do tipo $T$ maturadas, possuindo a capacidade de reconhecer os agentes patogênicos, ou seja, são habilitadas a detectar e identificar quase todo elemento não-próprio. Verifica-se, por fim, a afinidade comparando as cadeias escolhidas de forma aleatória com o conjunto de cadeias próprias. Sendo a afinidade superior a um limiar preestabelecido rejeitase a cadeia, categorizando-a como nãoprópria. Senão, coloca-se a cadeia no conjunto de detectores e essa será empregada nas classificações durante o monitoramento dos dados (Lima, 2013).

$\mathrm{Na}$ fase seguinte procura-se identificar alteração no comportamento das amostras, através do monitoramento dos dados. Se identificado, é realizada a classificação dessas mudanças utilizando o conjunto de detectores criados na fase de Censoriamento. Novamente, um elemento não próprio é detectado e classificado quando a afinidade entre as cadeias for superior a um limiar preestabelecido, como enfatiza Lima et al. (2016).

O critério conhecido como casamento, que pode ser perfeito ou parcial, é utilizado para avaliar a afinidade entre as cadeias e verificar que são iguais, semelhantes ou distintas.

Um casamento perfeito ocorre quando ambas cadeias são perfeitamente iguais, isto é, todas as posições das cadeias possuem os mesmos valores. Já o casamento parcial ocorre quando uma quantidade previamente determinada de posições entre as cadeias possui o mesmo valor. Essa quantidade é denominada taxa de afinidade (Castro, 2001).

O monitoramento da integridade estrutural (Structural Health Monitoring SHM) surge baseado na necessidade industrial de detectar de falhas em seu estado inicial para que assim se possa impedir sua propagação e se evitar prejuízos financeiros, humanitários e ambientais. Um SHM deve atender aos seguintes pontos: Aquisição e processamento de dados, validação e análise de sinais, identificação e caracterização de falhas, interpretação de 
mudanças adversas em uma estrutura e auxiliar a tomada de decisões (HALL, 1999).

O SHM baseado no algoritmo de seleção negativa possui o diferencial da estabilidade, podendo o sistema aprender com o tempo, e da plasticidade, a capacidade da continua aprendizagem com novos padrões incluídos mantendo o conhecimento anterior (Doebling et al., 1998).

Um SHM tendo como base o SIA, em particular o ASN (Forrest et al., 1994), tem seu diagnostico composto por duas fases: Censoriamento e monitoramento. Sendo que, no censoriamento é realizado um censo dos dados para a criação do conjunto de detectores e então o reconhecimento das falhas durante o monitoramento.

Alinhado com o exibido na Figura 3, a fase de censoriamento do SHM com base no ASN é dividida em dois módulos. A aquisição dos sinais é realizada no modulo de entrada, que compõe a bancada experimental. No modulo seguinte é gerado o conjunto de detectores próprios que será utilizado no monitoramento dos dados, esses são compostos por um grupo de sinais sem falhas na estrutura, isso é: Em situação estrutural normal. Esses dados recebem o nome de base-line, e servirão como parâmetro de comparação para identificação de falhas. Um sinal coletado da estrutura em uma situação de falha não apresentará afinidade satisfatória com a base-line, e assim é possível identificar a falha presente na estrutura. Já um sinal com a estrutura em situação normal ao ser comparado com o conjunto de detectores apresentará grande afinidade e não será identificada nenhuma falha (Lima, 2014).
Figura 3. Fluxograma da fase de censoriamento do SHM ASN.

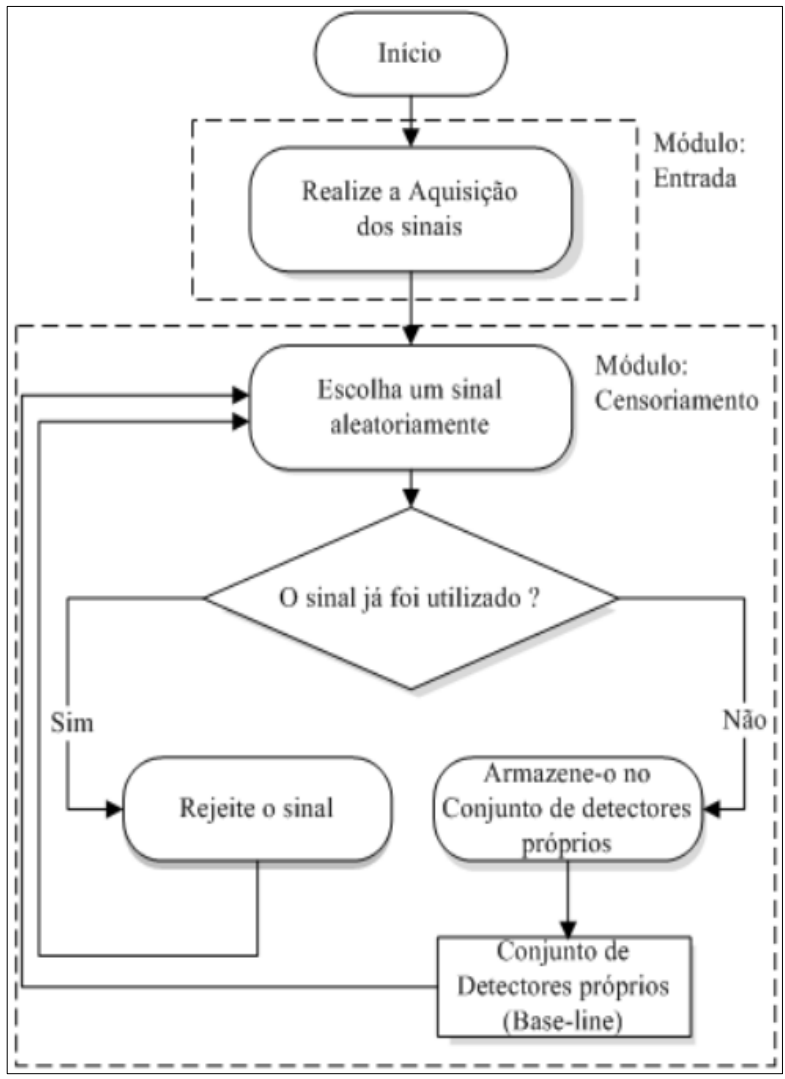

Fonte: (Lima, 2014).

No fluxograma da Figura 4 é ilustrada a fase de monitoramento, que também é dividida em duas etapas. Na etapa inicial é realizada a aquisição dos dados, enquanto que na última etapa a discriminação entre os sinais como sendo próprio/não-próprio.

No monitoramento analisa-se os dados em tempo real comparando-os com os detectores criados na fase anterior, buscando facilitar a tomada de decisão através do diagnóstico diferencial.

Feita a aquisição dos sinais executa-se o módulo de detecção onde os sinais em analise são comparados com os detectores próprios, buscando identificar o casamento entre os sinais. 
Figura 4. Fluxograma da fase de monitoramento do SHM ASN.

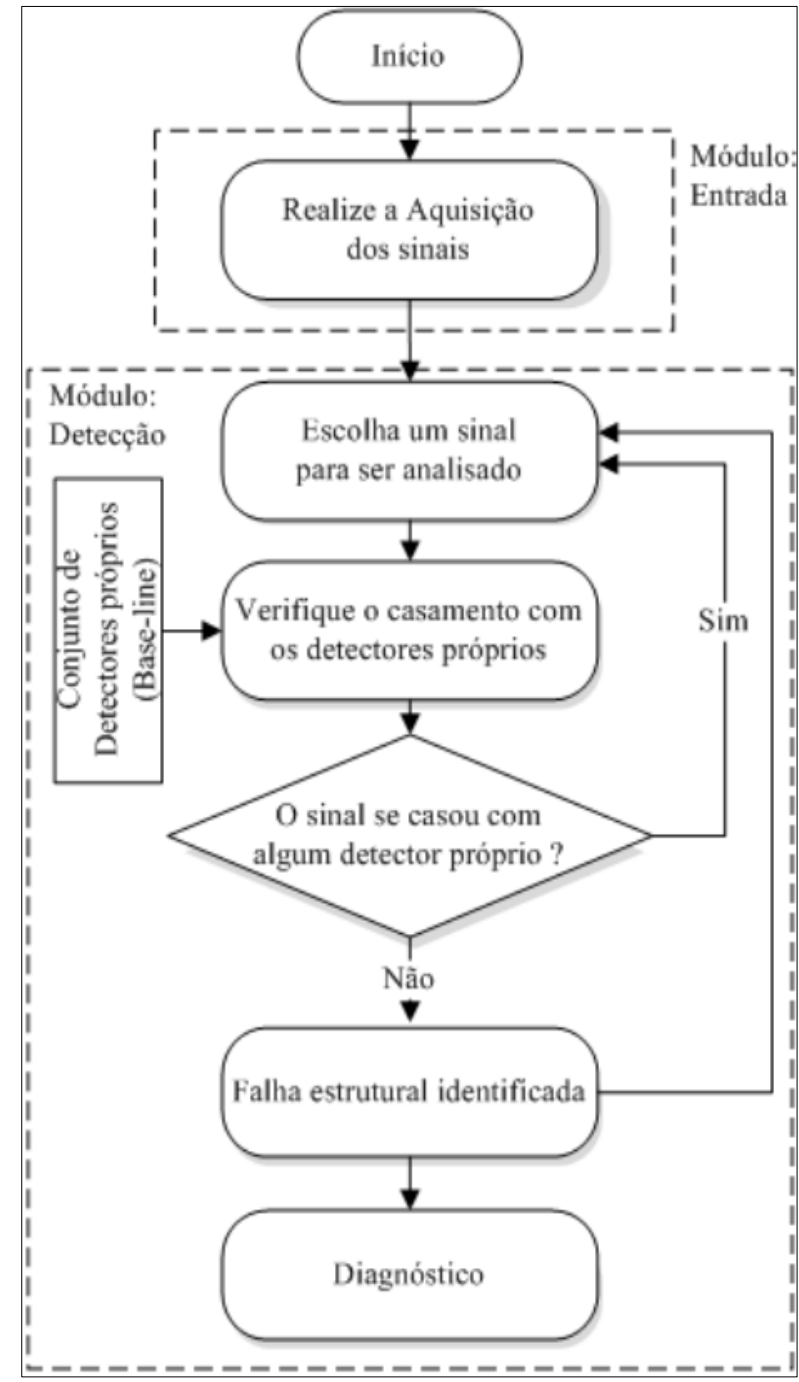

Fonte: (Lima, 2014).

Quando a afinidade é superior a taxa de afinidade definida os sinais em analise são categorizados como satisfatórios perante o conjunto de detectores e então classificados como sendo o sistema em situação normal (sem falhas). Caso a afinidade seja menor que a taxa de afinidade o sinal é classificado como uma anormalidade e uma falha estrutural é identificada.

Nesse trabalho foi utilizado o critério de casamento parcial proposto por Bradley e Tyrrell (2002), em conjunto com um desvio de $9 \%$ nos detectores.

\section{METODOLOGIA ADOTADA}

A coleta experimental desse trabalho foi realizada seguindo a norma ISO105341(1996). Essa, consiste na utilização de um microfone transladando horizontalmente em um tubo reto, rígido, de paredes não porosas, sem buracos ou fendas de secção transversal cilíndrica. A técnica da norma consiste emissão de uma onda senoidal por uma fonte sonora em uma das extremidades do tubo. Sendo a onda de frequência determinada, nesse caso propagando em fluido gasoso (ar). Na extremidade oposta é fixada a tampa com um furo em seu centro. Pela tampa é inserida a sonda com o microfone, que translada axial e internamente ao tubo, coletando as pressões. A Figura 5 esquematiza a montagem do experimento.

Figura 5. Desenho esquemático da montagem do experimento de bancada.

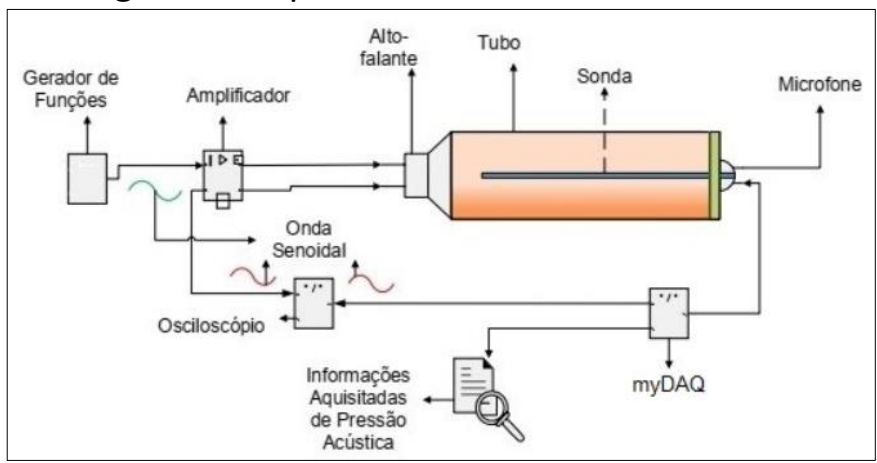

Fonte: Adaptada de (Outa, 2015, p.3).

Conforme exibido na Figura 5, o Gerador de Funções é ligado no Amplificador que, por sua vez, é conectado ao alto-falante e ao Osciloscópio. O microfone da sonda, que é conectado a placa de aquisição $m y D A Q$ da National Instruments (NI), realiza a coleta do sinal que é armazenado no Notebook, utilizando o National Instruments LabView Student Edition 2013 em conjunto com um algoritmo desenvolvido em Octave para a coleta.

A pressão sonora tanto na ponta interior quanto no exterior da sonda é a mesma. Este fato permite a utilização do microfone na extremidade exterior da sonda que capita a pressão de uma secção do tubo.

A frequência de corte é $2008 \mathrm{~Hz}$, determinada algebricamente em Gerges (2000, p. 290), para o diâmetro do tubo utilizado $(100 \mathrm{~mm})$; frequências acima deste 
valor não podem ser estudadas nessas condições.

Figura 6. Vista superior da bancada experimental.

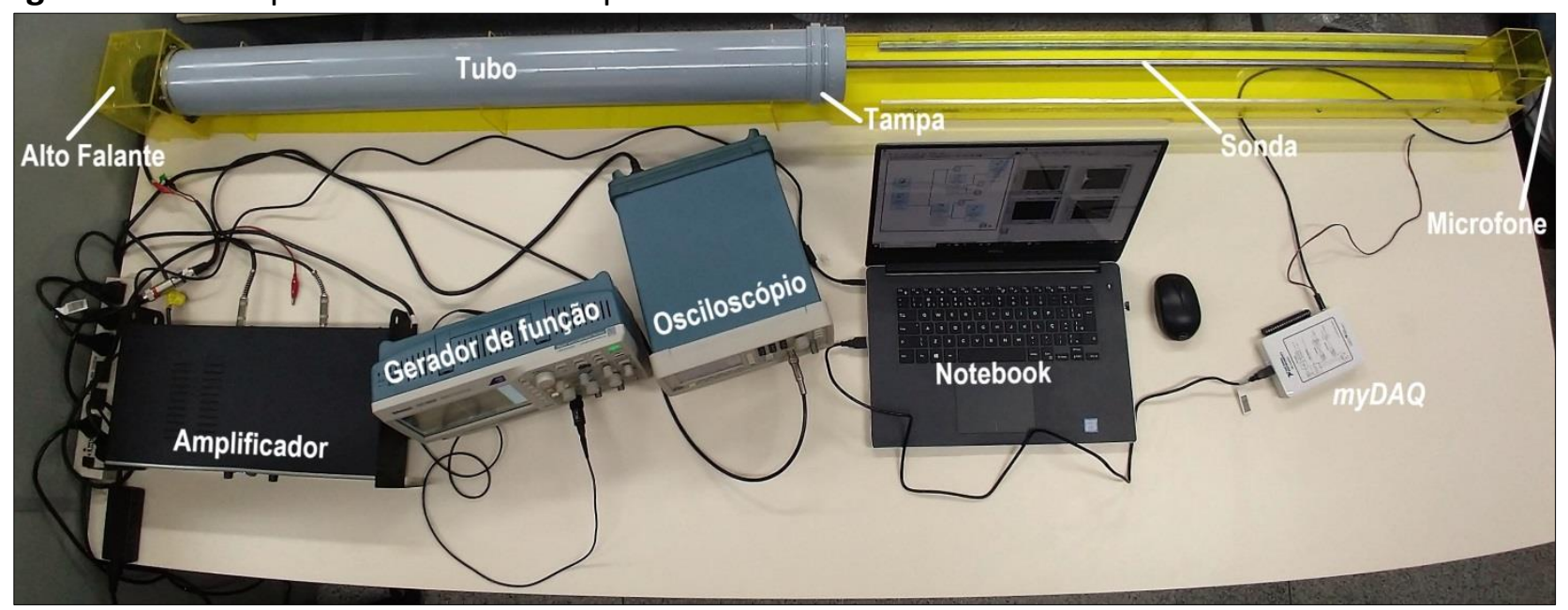

Fonte: (Feliciani Et Al., 2019).

A amplitude do sinal recebido pela fonte sonora deve respeitar a norma ISO10534-1 (1996), que determina que a onda emitida pela fonte seja preferencialmente $10 \mathrm{~dB}$ maior do que 0 maior ruído de fundo para a frequência em que se trabalha.

Na Figura 6 é possível visualizar a bancada experimental montada com os equipamentos utilizados na capitação do sinal.

\section{DESENVOLVIMENTO}

Quando uma onda acústica plana propagando em um meio I encontra uma superfície limite do seu meio uma onda refletida é formada no meio $I$, enquanto a onda transmitida no meio II, como mostra a Figura 7, diz (Kinsler et al., 1999).
Figura 7. Reflexão e transmissão de ondas.

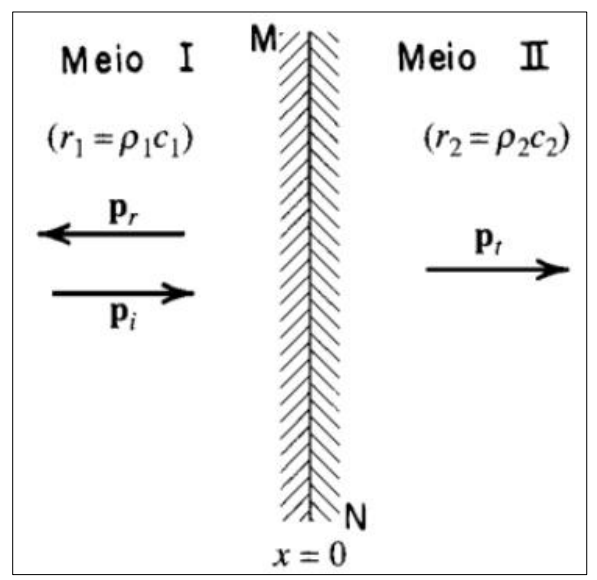

Fonte: Adaptada de (Kinsler et al., 1999, p. 151).

Na Figura 7 a membrana $\mathrm{MN}$ divide os meios I e II. A onda plana progressiva proveniente do Meio I incide normalmente a membrana propagando na direção positiva de $x$, sendo $x$ crescente no sentido do Meio II - a partir do referencial $(x=0)$ adotado na Figura 7.

Com a incidência, a onda refletida forma-se no meio I enquanto a transmitida no meio II (Gerges, 2000).

Com a propagação da onda incidente com uma amplitude adequada, internamente ao tubo há a formação da onda reincidente, conforme a teoria de transmissão e reflexão em dois meios (Kinsler et al., 1999). Verificase então que ocorre o principio de 
superposição de ondas no tubo. Ou seja, a somatória da onda incidente com a onda reincidente. A onda resultante da superposição recebe $\mathrm{o}$ nome de onda estacionaria.

Uma coleta experimental é composta pela soma das posições de 1 a $100 \mathrm{~cm}$ coletadas pela sonda que translada horizontalmente ao tubo coletando a pressão sonora de cada uma das posições. As coletas foram armazenadas em uma matriz com os dados experimentais.

\section{RESUTADOS}

A coleta experimental, do sistema com e sem falha, foi realizada em um tubo cilíndrico de 1 metro de comprimento em PVC. Seguindo os procedimentos expandidos na seção 4, foram coletados 10 sinais do tubo em situação estrutural normal e mais 3 sinais em situação de falha. Nesse caso, a falha no tubo foi simulada por um furo em sua superfície lateral, conforme exibido na Figura 8.

Figura 8: Vista superior do tubo com furo.

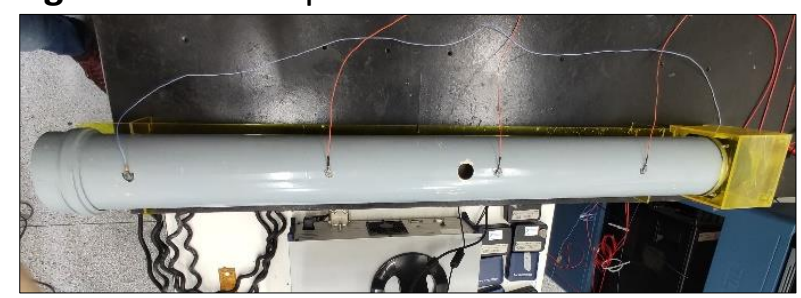

Fonte: Autoria própria.

A Figura 9 exibe um exemplo de sinal na frequência de $500 \mathrm{~Hz}$ coletado do tubo em situação estrutural normal.

Figura 9. Pressão sonora em situação estrutural normal - $500 \mathrm{~Hz}$.

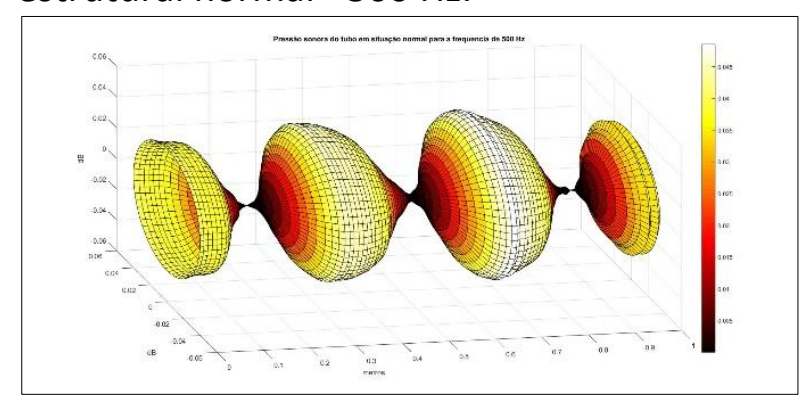

Fonte: Autoria própria.
Na Figura 9 visualiza-se a forma mais clássica de uma onda estacionaria, com pontos de pressão máximos e mínimos bem definidos.

A Figura 10 exibe uma das coletas experimentais realizadas no tubo em situação estrutural de falha.

Figura 10. Pressão sonora em situação estrutural de falha $-500 \mathrm{~Hz}$.

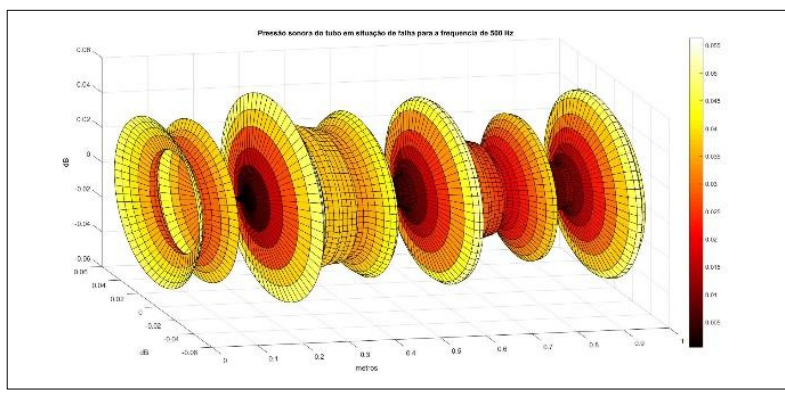

Fonte: Autoria própria.

Comparando a Figura 9 com a Figura 10 verifica-se que a falha causa uma interferência destrutiva nos pontos de pressão máximas da onda estacionaria. Para o caso estudado na frequência de $500 \mathrm{~Hz}$, os pontos de pressão mínima permanecem inalterados. Houve, também, uma redução de amplitude pelo sistema na condição de falha, devido também à perda de pressão, sendo identificada pelo SIA na detecção de falhas feita, como pode ser visualizado na Figura 11.

Após coletada a pressão sonora no interior do tubo, foi desenvolvido o SIA, e se iniciou o Módulo Censoriamento e posteriormente a Detecção do SHM. Assim, foi definida a base-line do sistema de forma a possibilitar a detecção de falhas. Para o caso dos resultados apresentados nesse trabalho $10 \%$ dos sinais normais, escolhidos aleatoriamente, foram selecionados para compor a base-line.

A Tabela 1 apresenta os resultados da operação do Sistema Imunológico Artificial (SIA) utilizando o Algoritmo de Seleção Negativa (ASN) (Forrest et al., 1994), por via acústica, para à frequência de $500 \mathrm{~Hz}$. Os dados encontrados na Tabela 1 se refere a 
comparação da respectiva coleta com a baseline de funcionamento do sistema.

Tabela 1. Detecção de falhas do SIA acústico utilizando $10 \%$ dos sinais normais para compor a base-line na frequência de $500 \mathrm{~Hz}$.

\begin{tabular}{c|c|c|c}
\hline Coleta & Afinidade & Variância & $\begin{array}{c}\text { Desvio } \\
\text { Padrão }\end{array}$ \\
\hline 1 & $82,00 \%$ & $9 * 10^{-7}$ & 0.00093218 \\
\hline 2 & $81,00 \%$ & $9 * 10^{-7}$ & 0.000957292 \\
\hline 3 & $80,00 \%$ & $8 * 10^{-7}$ & 0.000921402 \\
\hline 4 & $81,00 \%$ & $7 * 10^{-7}$ & 0.000837929 \\
\hline 5 & $83,00 \%$ & $8 * 10^{-7}$ & 0.000898317 \\
\hline 6 & $87,00 \%$ & $9 * 10^{-7}$ & 0.000929930 \\
\hline 7 & $80,00 \%$ & $8 * 10^{-7}$ & 0.000877635 \\
\hline 8 & $77,00 \%$ & $6 * 10^{-7}$ & 0.000796048 \\
\hline 9 & $81,00 \%$ & $7 * 10^{-7}$ & 0.000859609 \\
\hline 10 & $78,00 \%$ & $8 * 10^{-7}$ & 0.000889176 \\
\hline 11 & $12,00 \%$ & $4 * 10^{-7}$ & 0.000618987 \\
\hline 12 & $11,00 \%$ & $1 * 10^{-7}$ & 0.000264593 \\
\hline 13 & $11,00 \%$ & $1 * 10^{-7}$ & 0.000245606 \\
\hline
\end{tabular}

Fonte: (Feliciani Et Al., 2019).

Na tabela 1 , os sinais de 1 a 10 são dados coletados do tubo em situação estrutural normal de operação. Enquanto os dados de 11 a 13 referem-se a situação de falha estrutural.

O SIA foi capaz de identificar as 10 curvas do sistema em situação normal (1 a 10) e diferenciar as 3 em situação de falha (11 a 13), utilizando da afinidade. De acordo com Lima (2014), é recomendado o uso de até $70 \%$ dos sinais normais do sistema na fase de Censoriamento. No caso do algoritmo desenvolvido neste trabalho, como dito anteriormente, apenas $10 \%$ dos dados foram necessários para que o algoritmo alcançasse $100 \%$ de acerto - sendo o suficiente para o aprendizado o sistema.

Na tabela 1 , verifica-se que as coletas de 1 a 10 apresentaram afinidade satisfatória, ou seja, maior que a taxa de afinidade calculada (76,92\%), sendo essas identificadas pelo SAl como sendo da estrutura em situação normal. Já as curvas das coletas de 11 a 13 apresentaram afinidade com um erro de $84 \%$ em referência a taxa e afinidade calculada, o que justificou a categorização de situação estrutural de falha apontada pelo SIA.

Por fim, a Figura 11 foi gerada pelo ASN de forma a facilitar a compreensão humana do seu funcionamento. A figura é composta tela coleta 11 de pressão sonora, um sinal não-próprio. Os pontos em vermelho ilustram as partes do sinal que foram identificados como tendo afinidade satisfatória com a base-line.

Figura 11. Pressão sonora da coleta 11 com identificação da afinidade - $500 \mathrm{~Hz}$.

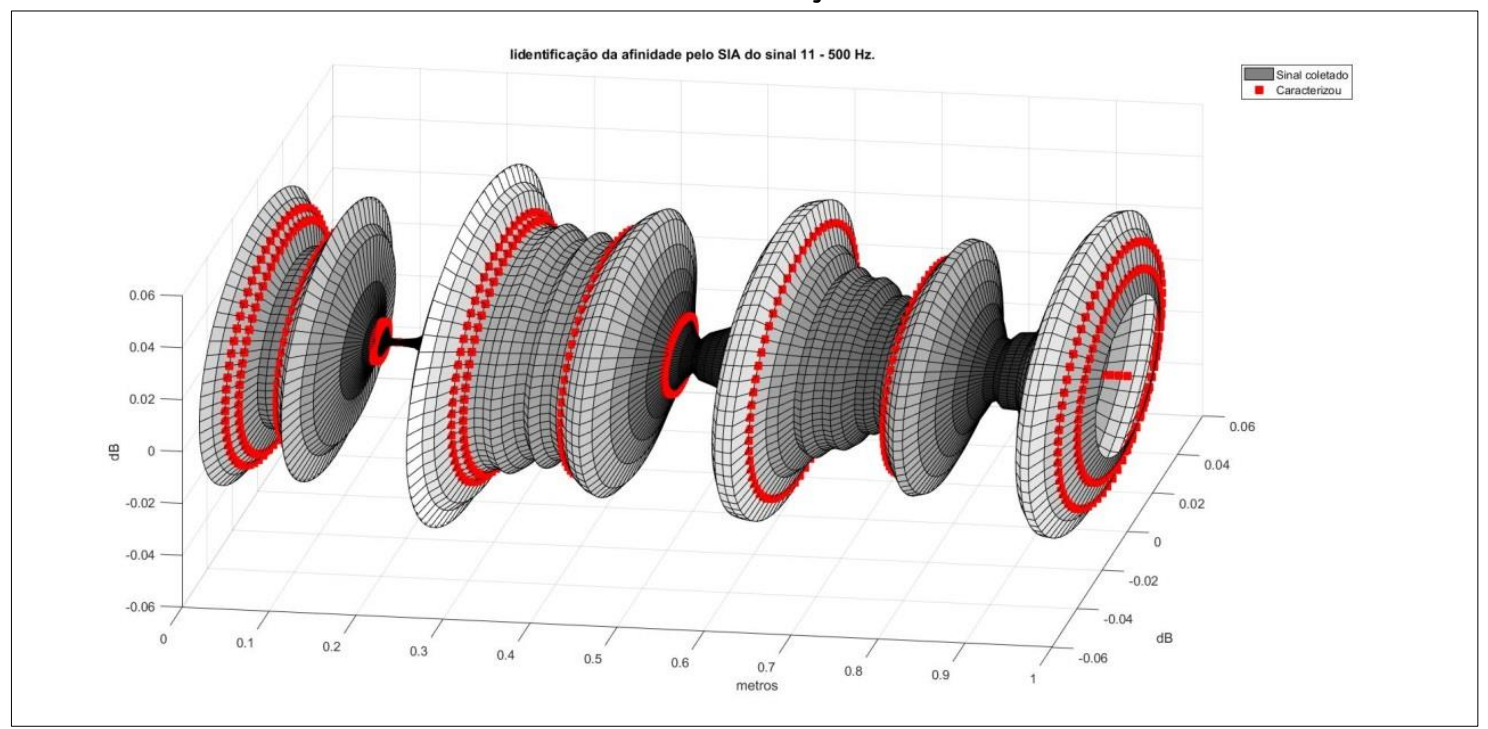

Fonte: Autoria Própria. 
Na Figura 11 é possível verificar os $12 \%$ de afinidade identificados pelo SIA e descrito na Tabela 1.

\section{CONSIDERAÇÕES FINAIS}

Os resultados obtidos pelo ASN foram: $0 \%$ de falsos positivos, $0 \%$ de equívocos cometido pelo sistema e taxa de acerto de $100 \%$. Exibindo sua robustez e eficácia na identificação e categorização de sinais próprios e não-próprios. Por tudo isso, o SIA mostrou ser uma interessante ferramenta alternativa para detecção de falhas em tubos, podendo ainda ser aplicado em sinais de aceleração do tubo para realizar-se a localização da falha.

A detecção de falhas também foi realizada para as frequências de 1000 e 2000 $\mathrm{Hz}$, além dos $500 \mathrm{~Hz}$ apresentados. Foi também realizada a detecção e localização de falhas, para as mesmas 3 frequências, utilizando via vibracional. Bem como a comparação entre os dois métodos. Todas as aplicações do SHM foram satisfatórias.

A metodologia proposta para a detecção de falhas é inédita para a área de SHM na utilização do meio acústico, sendo esse um ponto de diferencial deste trabalho.

\section{AGRADECIMENTOS}

Os autores agradecem a Fapesp (Proc.No.2018/16447-8 e Proc.No.2019/ 10515-4), ao Laboratório SISPLEXOS e a UNESP pelo suporte.

\section{REFERÊNCIAS}

De Castro, L. N. Engenharia imunológica: desenvolvimento e aplicação de ferramentas computacionais inspiradas em sistemas imunológicas artificiais. 2001. Tese de Doutorado (Engenharia Elétrica) Faculdade de Engenharia Elétrica e de Computação, Campinas - SP, 2001.

Doebling, S.W., Farrar, C. and Prime, M. A summary review of vibration-based damage identification methods, 1998. Vol. 30, pp. 91-105. https://doi.org/10.1177/0583102498030002 $\underline{01}$

Feliciani M., I.; Chavarette, F. R.; Outa, R. Caracterização de um tubo de impedância acústica via computação natural. Colloquium Exactarum. ISSN: 2178-8332, v. 11, n. 4, p. 62-72, 26 nov. 2019. DOI: 10.5747/ce.2019.v11.n4.e297.

https://doi.org/10.5747/ce.2019.v11.n4.e297

Forrest, S. A.; Perelson, A. L.; Cherukuri, R. Self-nonself discrimination in a computer. In: IEEE Symposium On Research In Security And Privacy, 1., 1994, Oakland. Proceedings... Oakland: IEEE, 1994. p. 202-212.

Franco, V. R.; Bueno, D. D.; Brennan, M. J.; Cavalini Jr., A. A.; Gonsalez, C. G.; Lopes JR., V. Experimental damage location in smart structures using Lamb waves approaches. In: Brazilian Conference on Dynamics, Control and Their Applications - DINCON, 2009. p. 14.

Gerges, S. N. Y. Ruído: fundamentos e controles. Florianopolis: NR Editora, 2000. $696 \mathrm{p}$.

Hall, S. R. The effective management and use of structural health data. In: International Workshop on Structural Health Monitoring, 1999. p. 265-275.

Kinsler, L. E. et al. Fundamentals of acoustics. 4. ed. Hoboken: John Wiley \& Sons, 1999. $548 \mathrm{p}$.

Lima, F. P. A. Análise de distúrbios de tensão em sistemas de distribuição de energia elétrica baseada em sistemas imunológicos artificiais. 2013. 169 f. Dissertação (Mestrado em Engenharia Elétrica) - Faculdade de Engenharia, Universidade Estadual Paulista UNESP, Ilha Solteira - SP, 2013.

Lima, F. P. A. Monitoramento e identificação de falhas em estruturas aeronáuticas e 
mecânicas utilizando técnicas de

computação inteligente. 2014. 72 p.

Dissertação (Mestrado em Engenharia

Mecânica) - Faculdade de Engenharia, Universidade Estadual Paulista - UNESP, Ilha Solteira - SP, 2014.

Lima, F. P. A. Diagnóstico de distúrbios de tensão em sistemas de distribuição baseado num sistema imunológico artificial com aprendizado continuado. 2016. Tese de Doutorado (Engenharia Elétrica) - Faculdade de Engenharia de Ilha Solteira, Ilha Solteira SP, 2016.

Outa, R.; Chavarette, F. R. Caracterização do experimento do tubo de impedância de um microfone. 2015. $4 \mathrm{f}$. Boletim Técnico da FATEC-SP | BT/39 | Junho / 2015. Disponível em:

http://bt.fatecsp.br/bulletins/show_article/9 90>. 